\title{
Prospects for the Determination of Star Orbits Near the Galactic Center
}

\author{
M. Jaroszyński \\ Warsaw University Observatory, Al. Ujazdowskie 4, 00-478 Warszawa,Poland \\ Visiting Scientists, Department of Astrophysical Sciences, Princeton University \\ e-mail: mj@sirius.astrouw.edu.pl
}

\begin{abstract}
We simulate the observations of proper motion of stars very close to the Galactic Center. We show that the speckle interferometry done with the Keck II telescope is accurate enough to obtain orbital parameters for stars with the period $P \sim 10$ y during $\sim 10$ seasons of astrometric observations made once a year. The determination of a single orbit will give central mass estimate with the typical uncertainty of the existing mass determinations based on velocity dispersion measurements. A much higher precision orbits will be measured in several years when Keck Interferometer becomes operational, and fainter stars are discovered even closer to Sgr $A^{*}$. Astrometry alone will provide accurate determination of $M / D^{3}$, where $M$ is the black hole mass and $D$ is the distance to the Galactic Center. If spectroscopic orbits of the stars are also measured then both: $M$ and $D$ will be precisely determined.
\end{abstract}

Subject headings: galaxies: black holes — galaxies: individual (Milky Way) 


\section{Introduction}

The proper motion studies of stars near the Galactic Center (Genzel et al. 1997; Eckart \& Genzel 1997; Ghez et al. 1998) show the astonishing accuracy of the astrometric observations in the near infrared $K$ band. The closest to Sgr A* studied star is at the projected distance $\sim 100$ mas (corresponding to $\sim 850$ AU at $8.5 \mathrm{kpc}$ ) from its position, and moves with the velocity $\sim 1400 \mathrm{~km} / \mathrm{s}$ in the plane of the sky. The radial velocities of some of the stars at $\geq 3^{\prime \prime}$ from Sgr A* are also measured (Genzel et al. 1996) and the comparison with the proper motion data shows that the velocity distribution is nearly isotropic. The published observations cover a relatively short span of time and the above authors use a statistical approach to find the mass in the central $\sim 0.01 \mathrm{pc}$ region around the Galactic Center. The present accuracy of the observations makes it possible to study the orbits of individual stars and derive the mass in the central part of the Galaxy by more direct methods, similar to those used in classical binary systems of stars.

Recently Salim \& Gould (1998) proposed the study of the orbits of individual stars in the vicinity of Sgr A* to get its distance. Such measurement may be based on the accumulation of astrometric data augmented by the radial velocity data. Salim \& Gould consider three stars with the smallest projected distances from Sgr A* which can be found in the Ghez et al. (1998) catalog. They investigate the accuracy of the Sgr A* distance estimate achieved after given observation time and its dependence on the actual orbit inclinations and periods of the chosen stars. They assume that the star positions will be obtained with the present accuracy (2 mas) and that the radial velocity will be measured with an error smaller than $50 \mathrm{~km} / \mathrm{s}$.

In this paper we address similar questions using a different approach. First we are interested in the accuracy of the determination of all orbit parameters and the accuracy of the determination of the central mass. We are also interested in determinations based on better astrometric accuracy and using fainter stars, which may in future be found closer to the Galactic Center. We do not use any particular stars with already measured proper motions, but rather simulate the orbits with given semimajor axis, not exceeding $\sim 10^{3} \mathrm{AU}$ and randomly chosen eccentricity and orientation in space. Similar approach has been used by Jaroszyński (1998b), in the study of the observability of relativistic effects in motion of stars close to Sgr A*. According to this paper only the relativistic motion of periastron would be measurable for orbits $\sim 1000$ AU in size and only if the accuracy of astrometric measurements is much higher than the present one, reaching the future capabilities of the Keck Interferometer (van Belle \& Vasisht 1998). We neglect the relativistic effects altogether using purely Newtonian star trajectories in our studies.

While the presence of the black hole in the Galactic Center has not been proven 
yet, and the existence of a dense cluster of some kind of dark matter here (Munyaneza, Tsikaluri, \& Violler 1998) has not been excluded, we assume that there is in fact a point mass in the Galactic Center. We adopt the central black hole mass estimate of Ghez et al. (1998), $M_{0}=2.6( \pm 0.2) \times 10^{6} \mathrm{M}_{\odot}$, and the distance to the Galactic Center, $D_{0}=8.5 \mathrm{kpc}$ for our simulations. Our analysis is aimed at finding the relative error in possible mass and distance estimates and does not depend critically on their exact values used for simulations. With the adopted distance and mass the angular size of 100 mas corresponds to 850 AU and to the orbital period of 15.4 y for an elliptical orbit of this semimajor axis.

In the next Section we consider the modeling of orbits based on astrometric observations alone. In Sec. 3 we consider fits based on the combined astrometric and radial velocity data. In Sec. 4 we estimate the number of stars in the close vicinity of Sgr A*, which may in future be used for black hole mass and its distance determination. The discussion follows in the last Section.

\section{Simulations of Astrometric Observations of Star Motions}

We consider stars on elliptic orbits with "true" semimajor axis $a_{0} \leq 10^{3} \mathrm{AU}$. The closest to Sgr A* star with measured proper motion (Ghez et al. 1998) is at the projected distance 114 mas, so its 3D distance $r \geq 969$ AU and the semimajor axis of its orbit must be greater than $485 \mathrm{AU}$. (It can be much greater, of course.) We are also interested in faint stars ( $K \leq 17$, or fainter), which may in future be found at similar or smaller distances from the Galatic Center. We postpone the discussion of the probability of finding such stars until Sec. 4.

We assume the accuracy of the relative position measurements to be constant in time and the observations to take place once a year, as has been the practice until now. According to Ghez et al. (1998) the uncertainty of the relative position measurements for bright $(K \leq 15)$ stars near Galactic Center is typically $\sim 2$ mas, which corresponds to $17 \mathrm{AU}$. The proper velocity measurements are less accurate for fainter stars, with uncertainty doubling at each 2 magnitude interval. It suggests indirectly, that for faint stars $(K \leq 17)$ the uncertainty in the relative position amounts to $\sim 4$ mas, or 34 AU. These are the typical numbers we are using in our numerical experiments. In the future the Keck Interferometer (van Belle and Vasisht 1998) will achieve $\sim 20 \mu$ as accuracy in astrometric mode, corresponding to $0.17 \mathrm{AU}$. This number is another characteristic value, which can be used for simulations.

We introduce a Cartesian coordinate system $\left(x_{0}, y_{0}\right)$ in the orbital plane with the origin 
at the position of the black hole and the $x_{0}$ axis pointing toward the periastron. The "true" orbit is given as:

$$
\frac{2 \pi}{P_{0}}\left(t-t_{0}\right)=u-e_{0} \sin u ; \quad x_{0}=a_{0}(\cos u-e) ; \quad y_{0}=b_{0} \sin u
$$

where $P_{0}$ is the orbital period, $t_{0}$ - time of the passage through the periastron, $a_{0}$ and $b_{0}$ are the major and minor semiaxes of the ellipse, $e_{0}$ is its eccentricity, and $u$ - eccentric anomaly. The orientation of the ellipse in space is given by the three angles (inclination $i_{0}$, position angle of the line of nodes in the sky $\Omega_{0}$, and the angle of the periastron measured from the ascending node of the orbit $\left.\omega_{0}\right)$. The position of the star in the sky is obtained after the projection of its position in space, which is given as:

$$
\mathbf{r}(t)=x_{0}(t) \mathbf{e}_{x}+y_{0}(t) \mathbf{e}_{y}
$$

where $\mathbf{e}_{x}$ and $\mathbf{e}_{y}$ are the $3 \mathrm{D}$ unit vectors along $x_{0}$ and $y_{0}$ axes.

Our approach is a Monte Carlo simulation of synthetic data sets (e.g. Press et al. 1987). Usually one has a model fitted to the real data and is interested in the confidence limits on the estimated parameters. One of the possible way of doing it is to take the fitted parameters as true and simulate the sets of observations of the system assuming that the model is a good representation of the system. In our case the known orbit parameters allow the calculation of the accurate star position on the sky at any time. The measured positions are in error. With the estimated uncertainty in position measurements $\sigma$ we assume the measured position to be normally distributed:

$$
p(X) d X=\frac{1}{\sqrt{2 \pi} \sigma} \exp \left(-\left(X-X_{0}\right)^{2} / 2 \sigma^{2}\right) d x
$$

where $X$ can be any of the two measured coordinates of the star on the sky, and $X_{0}$ is its "true" value. We draw randomly the simulated positions of the star from the probability distribution and obtain the synthetic data set. Repeating the procedure we get many such sets. Fitting models to these simulated observations we obtain different sets of model parameters scattered around the original values. The scatter in these fitted parameters is a good measure of the confidence limits of the original fit. Thus starting with an orbit of known parameters and simulating many synthetic data sets with the same uncertainty $\sigma$, we can learn about a likely quality of the fitted model. In particular we can estimate the typical errors of the fit.

The mass of the black hole is related to the orbital parameters through the Kepler's law:

$$
M=\frac{4 \pi^{2} a^{3}}{\mathrm{G} P^{2}}
$$


where $G$ is the gravitational constant. In this Section we assume that the distance to the Galactic Center is known and equal to $D_{0}=8.5 \mathrm{kpc}$, so the directly measurable angular sizes are equivalent to corresponding linear sizes. In general the quantity which can be estimated from the astrometric data alone is the ratio $M / D^{3}$. In our simulations of star orbits we use $M_{0}=2.6 \times 10^{6} M_{\odot}$ for the value of the black hole mass. The mass estimated from the models fitted to simulated data is scaterred around $M_{0}$. For different central mass values of some of the fitted parameters would scale, but the procedure would remain the same.

We find the model parameters using the least square minimization of the expression:

$$
\chi^{2}=\sum_{j=1}^{N} \frac{\left(\mathbf{X}_{j}-\mathbf{X}\left(t_{j} ; a, e, P, i, \Omega, \omega, t_{0}\right)\right)^{2}}{\sigma^{2}}
$$

where $\mathbf{X}_{j}$ is the "measured" position of the star at the time $t_{j}$ and $\mathbf{X}\left(t_{j} ; a, e, P, i, \Omega, \omega, t_{0}\right)$ is the position resulting from a model with the given parameters and calculated for the same instant of time.

The semimajor axis $a_{0}$ of the "true" orbit serves as a main parameter of our simulations. Our study shows that the quality of the fits depends mostly on this parameter (and on the "true" period, since the two are related). For practical reasons we limit the number of iterations in the procedure finding the minima of $\chi^{2}$. A deeper analysis of the fitting shows a weak dependence of its success on the orbit eccentricity, showing that the cases with $e \approx 1$ are relatively more difficult. We neglect this fact in our simulations, which means, that the orbits having the above property are slightly underrepresented among successful fits. For a given $a_{0}$ we choose the eccentricity $0 \leq e_{0} \leq 1$ as a random number. The $\cos i_{0}, \Omega_{0}$, and $\omega_{0}$ are also given random values to guarantee the isotropic distribution of orbits orientation and position of the periastron. The time of periastron passage has no physical meaning (any properties of the motion depend on $t-t_{0}$ only) so we choose it at random from the range $0-P$.

We assume observations to take place in a randomly chosen day of June and to be repeated through $N$ seasons. The basic number of observations we consider is $N=10$, but we also make fewer simulations for other numbers. For each synthetic data sets $\left\{\mathbf{X}_{j}\right\}$ we find a model, starting the fitting procedure from the "true" values of the parameters. Since we expect the parameters fitted to the scattered data to be close to the "true" parameters, this starting point seems to be the best. If the fit converges, and if the obtained minimal $\chi^{2}$ is smaller than the tabularized value for given number of the degrees of freedom and required confidence, we include the parameters of the model to the sample. Otherwise we neglect them, but we keep the track of such unsuccessful fits. 
The results of our simulations are shown in Figs 1,2. In the upper panel of Fig. 1 we show the ratio of the median value of the fitted semimajor axis $a$ to its "true" value $a_{0}$. We also draw the lines showing the region including $68 \%$ of the sample points. Since the accuracy of position measurement is constant, the relative errors in fitted values of $a$ are larger for smaller orbits. The opposite can be said of the accuracy of the period estimates, which become more accurate when the total span of observations becomes longer than the orbital period. That means increasing accuracy for smaller orbits. The eccentricity is

related to the orbit shape and can be better estimated for large orbits. In Fig.2 we show the result for mass estimation based on the estimation of the orbital parameters. In this plot we see that the estimates for small orbits become less accurate. Even if there are faint stars on close orbits near the Galactic Center, the speckle interferometry with the Keck telescope, with the position uncertainty of $\sim 2$ mas $(17 \mathrm{AU})$ is not sufficient to give mass estimates better than obtained with the existing methods. For the stars on large $(\sim 10$ y) orbits the "once a year" strategy seems promising, but requires several years of data acquisition.

\section{Simulations of Astrometric and Radial Velocity Observations}

The measurements of the radial velocities for some of the Galactic Center stars with measured proper motions have been done (Genzel et al. 1996) with the accuracy of $\sigma_{v}=30 \mathrm{~km} / \mathrm{s}$. These stars are rather far from the center (at projected distance $\geq 3^{\prime \prime}$ ), but similar measurements for stars closer to the center, in the crowded field of view, may be possible in the future. We optimistically assume that the same accuracy of radial velocities will be possible for the stars closer to the center.

With radial velocities measured and orbits determined from the proper motion study, it is possible to estimate the distance $D$ to the source (Salim \& Gould 1998). We use now $\alpha_{0}$ - the angular measure of the orbit semimajor axis as an independent parameter. (One has $a_{0} \equiv D \alpha_{0} ; b_{0} \equiv D \beta_{0}$.)

The velocity components of a star moving on an elliptic orbit are:

$$
v_{0 x}=-\frac{2 \pi D \alpha_{0}}{P_{0}} \frac{\sin u}{1-e \cos u} ; \quad v_{0 y}=+\frac{2 \pi D \beta_{0}}{P_{0}} \frac{\cos u}{1-e \cos u}
$$

where we use the reference frame of equation (1). The velocity vector in space is given as:

$$
\mathbf{v}_{0}=v_{0 x}(t) \mathbf{e}_{x}+v_{0 y}(t) \mathbf{e}_{y}
$$

and its component along the line of sight can (in principle) be measured. The parameter $V_{0} \equiv 2 \pi D \alpha_{0} / P_{0}$ measures the amplitude of the velocity and can be independently fitted 
using the radial velocity data. The model of the orbit including radial velocities has eight parameters and can be fitted after the minimization of the expression

$$
\chi^{2}=\sum_{j=1}^{N} \frac{\left(\mathbf{X}_{j}-\mathbf{X}\left(t_{j} ; \alpha, e, P, i, \Omega, \omega, V, t_{0}\right)\right)^{2}}{\sigma^{2}}+\sum_{j=1}^{N_{v}} \frac{\left(v_{j}-v\left(t_{j} ; \alpha, e, P, i, \Omega, \omega, V, t_{0}\right)\right)^{2}}{\sigma_{v}^{2}}
$$

where $N_{v}$ is the number of radial velocity measurements, $v_{j}$ is the $\mathrm{j}$-th measured radial velocity, and $v\left(t_{j} ; \alpha, e, P, i, \Omega, \omega, V, t_{0}\right)$ is the radial velocity at the instant $t_{j}$ resulting from the model with given parameter values.

With the velocity measured independently, the central mass and its distance can be estimated:

$$
\begin{gathered}
M=\frac{P V^{3}}{2 \pi \mathrm{G}} \\
D=\frac{V P}{2 \pi \alpha}
\end{gathered}
$$

where all the variables in the RHS are given by the fit. In Figure 3 we show the results of our simulations including radial velocity measurements. The results of the velocity fitting, mass estimate, and distance estimate are shown. As can be seen in the plots, the relative error in velocity fit becomes smaller for smaller orbits and the same can be said about the mass estimation. Ten astrometric observations with accuracy of $\sim 2$ mas with 5 radial velocity measurements done in the period of $\sim 10$ years are sufficient to give the distance to the Galaxy Center with a accuracy better than $5 \%(1 \sigma)$ for large enough orbits ( $\geq 200$ AU).

\subsection{Accuracy of parameter fitting}

The improvement of interferometric equipment is expected to give much better accuracy of position measurements, reaching $\sigma \sim 20 \mu$ as (van Belle \& Vasisht 1998) in the case of the Keck Interferometer. We investigate the influence of the position accuracy on the expected errors in fitted parameters, for the whole range of $\sigma$ from $20 \mu$ as to 4 mas ( 0.17 to $34 \mathrm{AU})$. We repeat our simulations for several values of $\sigma$ and several values of the ellipse semimajor axis, using the same "observational strategy" as above. "Observations" of each orbit of a star are simulated many times. For every simulation we get a set of

estimated orbit parameters. We define the scatter in the estimated values of a parameter $p$ (where $p \in\left\{a, e, P, t_{0}, i, \Omega, \omega, V\right\}$ ) of a given orbit as

$$
\delta p=\left(p_{+}-p_{-}\right) / 2
$$

where $16 \%$ of the estimated values of $p$ are greater than $p_{+}$, another $16 \%$ are below $p_{-}$, and the remaining $68 \%$ are between them. The scatter in the estimation of the parameter $p$ for 
all the orbits of the same size $a_{0}$, observed with the same position accuracy $\sigma$ is given as:

$$
\Delta p=<\delta p>
$$

In Fig. 4 we show the scatter in the fitted parameters. The ratio $\sigma / a_{0}$ is a good estimate of the deformation introduced to the visual orbit, so we use it as the abscissa for our plots. In the left column we show the results for simulations based on astrometric measurements alone. In the right column the typical errors in the orbit elements fitting are shown for combined astrometric and radial velocity synthetic data. It can be seen, that the radial velocity data, which has fixed accuracy in our simulations, can improve the fitting procedure in case of poor astrometric accuracy.

We consider also the accuracy of mass and distance determinations. In Fig. 5 we display the typical uncertainty in mass estimation based on two methods and the results for the distance determination. Again, the increased astrometric accuracy does not help much the determinations based on radial velocity data.

\section{Possibility of Observing Stars Closer to the Center}

The central star cluster (Genzel et al. 1996, 1997) is the dominating stellar component within $\sim 10^{2}$ pc from the Galaxy Center. The density distribution follows the softened isothermal sphere model

$$
n(r)=\frac{n_{c}}{1+\left(r / r_{c}\right)^{2}}
$$

with the core radius $r_{c}=0.22 \mathrm{pc}(5.33 \mathrm{arcsec})$. Projection along the line of sight gives the surface concentration of stars:

$$
\mathcal{N}(R)=\pi n \frac{r_{c}^{2}}{\sqrt{r_{c}^{2}+R^{2}}} \equiv \mathcal{N}_{K} \frac{r_{c}}{\sqrt{r_{c}^{2}+R^{2}}}
$$

where $R$ is the distance from the black hole measured in the plane of the sky, and $\mathcal{N}_{17}=20 \operatorname{arcsec}^{-2}$ for stars brighter than $K=17^{m}$. In the whole region of interest to us $\left(R \ll r_{c}\right)$ the surface density of stars belonging to the cluster core is constant.

According to Ghez et al. (1998) the sample of stars in their proper motion studies constitutes a distinct cluster with the core radius $r_{c 1}=0^{\prime \prime} 3(\sim 0.01 \mathrm{pc})$ and the peak surface density of $\mathcal{N} \approx 15 \operatorname{arcsec}^{-2}$. Since the central surface densities of both clusters are similar the volume density in the smaller one is $\sim r_{c} / r_{c 1}$ times larger than the volume density in the core of the background cluster. Thus approximately half of the stars that could be seen very close to Sgr $\mathrm{A}^{*}$ on the sky are indeed the stars very close to the center in 3D. 
The proper motion sample of stars (Ghez et al. 1998) is not complete and probably cannot be used as a tracer of the general population of stars in the very center of the Galaxy. On the other hand we expect that the future proper motion studies of the Galaxy Center will employ similar selection criteria, so the resulting samples will have similar space distribution.

We are interested in the density of the observable stars which are well inside the core. The observability of sources is limited by the spatial resolution of the interferometer at given limiting brightness. For the spatial resolution $d$ of the interferometer the density of stars should not be too big:

$$
\mathcal{N}(2 d)^{2} \leq 1 \quad \Rightarrow \quad \mathcal{N}_{\max } \sim \frac{1}{4 d^{2}} \sim 10^{4} \operatorname{arcsec}^{-2}
$$

where we adopt $d \approx 5$ mas as the resolution of the Keck Interferometer.

The combined surface star density in both clusters for $K \leq 17^{m}$ is $\mathcal{N}_{17} \approx 35 \operatorname{arcsec}^{-2}$ (Genzel et al. 1997; Alexander \& Sternberg 1998; Ghez et al. 1998). The maximal surface density of stars $\mathcal{N}_{\max }$ is $\sim 300$ times larger. The integral luminosity function for the Galaxy Center has the slope $\beta=0.875$ at $K=17^{m}$ (Blum et al. 1996). This slope flattens for stars less massive than $\sim 0.7 M_{\odot}$ (Holtzman et al. 1998), which corresponds to $K \approx 21^{\mathrm{m}}$ (Alexander \& Sternberg 1998) if we adopt the extinction $A_{K}=3.5$ (Blum et al. 1996) to the Galactic Center. Rescaling to $21^{m}$ in $K$ we have $\mathcal{N}_{21} \approx 25 \mathcal{N}_{17}$, much less than the maximal surface density introduced above. Thus the possibility of finding faint stars close to the Galactic Center is limited by their volume density and not by the limited resolution of the Keck Interferometer.

Using the same luminosity function to the proper motion sample of Ghez et al. (1998) we find that there should be $\sim 25$ times more stars with $K \leq 21$ and with measurable proper motions. Some of them may be located closer to the black hole than the stars already observed. For such faint stars the Keck Interferometer operating in the imaging mode will have position accuracy of $\sim 3$ mas. The highest accuracy for astrometry $(\sim 20 \mu \mathrm{as})$ will be possible for stars much brighter, $K \leq 17.6$ (van Belle \& Vasisht 1998).

\section{Discussion and Conclusions}

We have investigated elliptical orbits of stars in the Newtonian potential of a point mass. According to Jaroszyński (1998b) the periastron motion of the orbit due to the relativistic effects may be measurable for small enough orbits $\left(a \lesssim 10^{3}\right.$ AU). Similar

effect, but of different sign, may be caused by the significant amount of matter distributed 
continuously around the central black hole. The gravitational lensing in the vicinity of Sgr A* (Jaroszyński 1998a) may deform a part of the visual orbit if the observer is close to the orbital plane and when the star is behind the central mass. All these effects are easy to account for and can be introduced to the model. We neglect them here, since they have no significant influence on the accuracy of parameters fitting or mass and distance estimates.

Since we limit the number of iterations in the procedure finding $\chi^{2}$ minima, we have investigated the influence of this fact on the estimated errors in fitted parameters. We are interested mostly in the semimajor axis, period and mass estimates. As our analysis shows, the number of iterations necessary for the fitting procedure to converge increases with increasing orbit eccentricity. We have performed extra calculations for orbits with the true semimajor axis $a_{0}=800 \mathrm{AU}$, eccentricity changing from $e_{0}=0.01$ to 0.99 and random orientation in space, simulating the astrometric observations of the moving stars with the position accuracy of $\sigma=17 \mathrm{AU}$. The calculations show that the convergence is reached in $\sim 98 \%$ of the cases for $e_{0} \leq 0.8$ and falls to $\sim 90 \%$ for $e_{0}=0.99$. The quality of the fits, as measured by the value of $\chi^{2}$ does not depend on the orbit eccentricity and the same is true about the scatter in the estimated orbital period. The relative error in the estimated semimajor axis is up to $\sim 2$ times larger for highly eccentric orbits $\left(e_{0} \geq 0.8\right)$ as compared to lower eccentricity orbits. Since this group of the orbits is underrepresented, the error is underestimated, but only slightly. (An analysis taking into account the adequate number of high eccentricity orbits would give $\lesssim 1.01$ times larger error estimates for semimajor axis and $\lesssim 1.03$ times larger scatter in mass estimates. Calculations with doubled number of allowed iterations confirm this reasoning.) Similar investigation shows, that the orbit inclination has negligible influence on the accuracy of the estimated semimajor axis, period, or central mass.

The investigation of the proper motion of stars at distances $\lesssim 10^{3}$ AU from Sgr A* can provide a robust test of the existence of a black hole there. If the mass is indeed in the form of a black hole, and the amount of mass distributed continuously is insubstantial, than the stars should move on elliptical orbits and the rate of periastron motion should agree with the mass estimated from the orbit size and period. Each orbit directly probes the distribution of mass at distances $(1-e) a \leq r \leq(1+e) a$. Knowing few orbits one may cover a substantial range in distances from the central mass. For such a test faint stars, which may be found closer to the center than already observed relatively bright stars with $K \leq 17$, can be used. The stars closer to the center have shorter periods, so their orbits can be found in shorter time. The increased accuracy of the astrometric position measurements will give very accurate orbits for bright stars. This may eventually serve as a test of point mass hypothesis at distances $\gtrsim 10^{3} \mathrm{AU}$. 
The measurement of radial velocities allows for the measurement of the distance to Sgr $\mathrm{A}^{*}$ (Salim \& Gould,998) and the absolute estimate of its mass. With the present accuracy of astrometry (2 mas) and spectroscopy $(30 \mathrm{~km} / \mathrm{s})$ in $K$ band, and with "observational strategy" adopted in our study, the most promising for the distance estimate are the orbits with $a \approx 600 \mathrm{AU}$, which would give $\sim 3 \%$ accuracy of the measurement in 10 years. Better accuracy and for slightly larger orbits can be obtained after longer time (Salim \& Gould 1998). The mass estimate based on the radial velocity becomes more accurate for smaller orbits. Their investigation is so challenging observationally, that it is probably better to use large orbits and wait longer for the results.

Special thanks are due to Bohdan Paczyński for suggesting the topic of this research, interest in its progress and kind hospitality during my stay at the Department of Astrophysical Sciences, Princeton University. This project was supported with the NASA grant NAG5-7016 to B. Paczyński and the Polish KBN grant 2P03D-012-12 to M. Jaroszyński. 


\section{REFERENCES}

Alexander, T., \& Sternberg, A. 1998, astro-ph/9811038

Blum, R. D., Sellgren, K., \& DePoy, D. L. 1996, ApJ, 470, 864

Eckart, A. \& Genzel, R. 1997, MNRAS, 284, 576

Genzel, R., Eckart, A., Ott, T., \& Eisenhauer, F. 1997, MNRAS, 291, 219

Genzel, R., Thatte, N., Krabbe A., Kroker, H., \& Taconi-Garman, L. E. 1996, ApJ, 472, 153

Ghez, A. M., Klein, B. L., Morris, M., \& Becklin, E. E. 1998, ApJ, 509, 678

Holtzman, J. A., et al. 1998, AJ, 115, 1946

Jaroszyński, M. 1998a, Acta Astronomica, 48, 413

Jaroszyński, M. 1998b, Acta Astronomica, 48, 653

Munyaneza, F., Tsiklauri, D., \& Violler, R. D., 1998, ApJ, 509, L105

Press, W. H., Teukolsky, S. A., Vettering, W. T., \& Flannery, B. P. 1988, Numerical Recipes: the Art of Scientific Computing (Cambridge: Cambridge University Press)

Van Belle, G. T. \& Vasisht, G. 1998, The Keck Interferometer: Science Requirements Document, JPL D-15477, (http://huey.jpl.nasa.gov/keck/) 
Fig. 1. - The median values of the ratio of the fitted orbit semimajor axis to the "true" value of this parameter $a / a_{0}$ (top panel), the median value of the difference $e-e_{0}$ (middle panel), and the ratio of the fitted period to its "true" value $P / P_{0}$ (lower panel) are drawn as functions of the "true" value of the semimajor axis $a_{0}$ with heavy lines. The thin lines encompass the region containing $68 \%$ of the fitted parameters values. Two cases $(\sigma=17$ AU and $\sigma=34 \mathrm{AU}$ ) are plotted. The results are obtained for $N=10$ "observations" taken once a year. The semimajor axis of 1000 AU corresponds to 118 mas in the sky for the galactocentric distance of $8.5 \mathrm{kpc}$.

Fig. 2. - The ratio of the fitted mass to the black hole mass used in simulations, $M / M_{0}$, shown as a function of the "true" semimajor axis $a_{0}$. The distance to the Galactic Center is assumed to be $D_{0}=8.5 \mathrm{kpc}$. The conventions follow Fig. 1, and the number of "observations" is $N=10$. The semimajor axis of 1000 AU corresponds to 118 mas in the sky for the galactocentric distance of $8.5 \mathrm{kpc}$.

Fig. 3.- The ratios of the fitted velocity (upper panel), mass (middle), and distance (lower) to the corresponding "true" values used in simulations. The mass estimate is based on the fitted velocity and period without postulating any value of the distance. The conventions follow Fig. 1. The simulations use $N=10$ astrometric and $N_{\mathrm{v}}=5$ spectroscopic "observations". The angular size of the orbits depends on the actual distance to Sgr A*; for $D_{0}=8.5 \mathrm{kpc}$ the semimajor axis of $1000 \mathrm{AU}$ corresponds to 118 mas.

Fig. 4.- The scatter in fitted orbital parameters as a function of relative accuracy in position measurements $\sigma / a_{0}$. Each panel shows the scatter in one parameter labeled along the y-axis. In the left column the results based on astrometric "observations" alone $\left(N=10, N_{\mathrm{v}}=0\right)$ are shown and in the right column the results related to combined astrometric and radial velocity "observations" ( $\left.N=10, N_{\mathrm{v}}=5\right)$ are displayed. Simulations use two values of the true semimajor axis $a_{0}=800 \mathrm{AU}$ (solid lines) and $200 \mathrm{AU}$ (dotted lines). While the assumed accuracy of the position measurement changes substantially we keep the assumed accuracy of radial velocity measurements constant.

Fig. 5.- The scatter in the estimated mass of the central black hole and in the estimated distance to the Galaxy Center. The upper panel shows the scatter in the mass estimate based on astrometry alone, under the assumption that the distance to the source is known. The middle panel shows the scatter in mass estimate, which is based on radial velocity and astrometric measurements and is independent of the distance to the source. The lower panel shows the scatter in the distance to the source based on the radial velocity measurements. Conventions follow Fig. 4. 


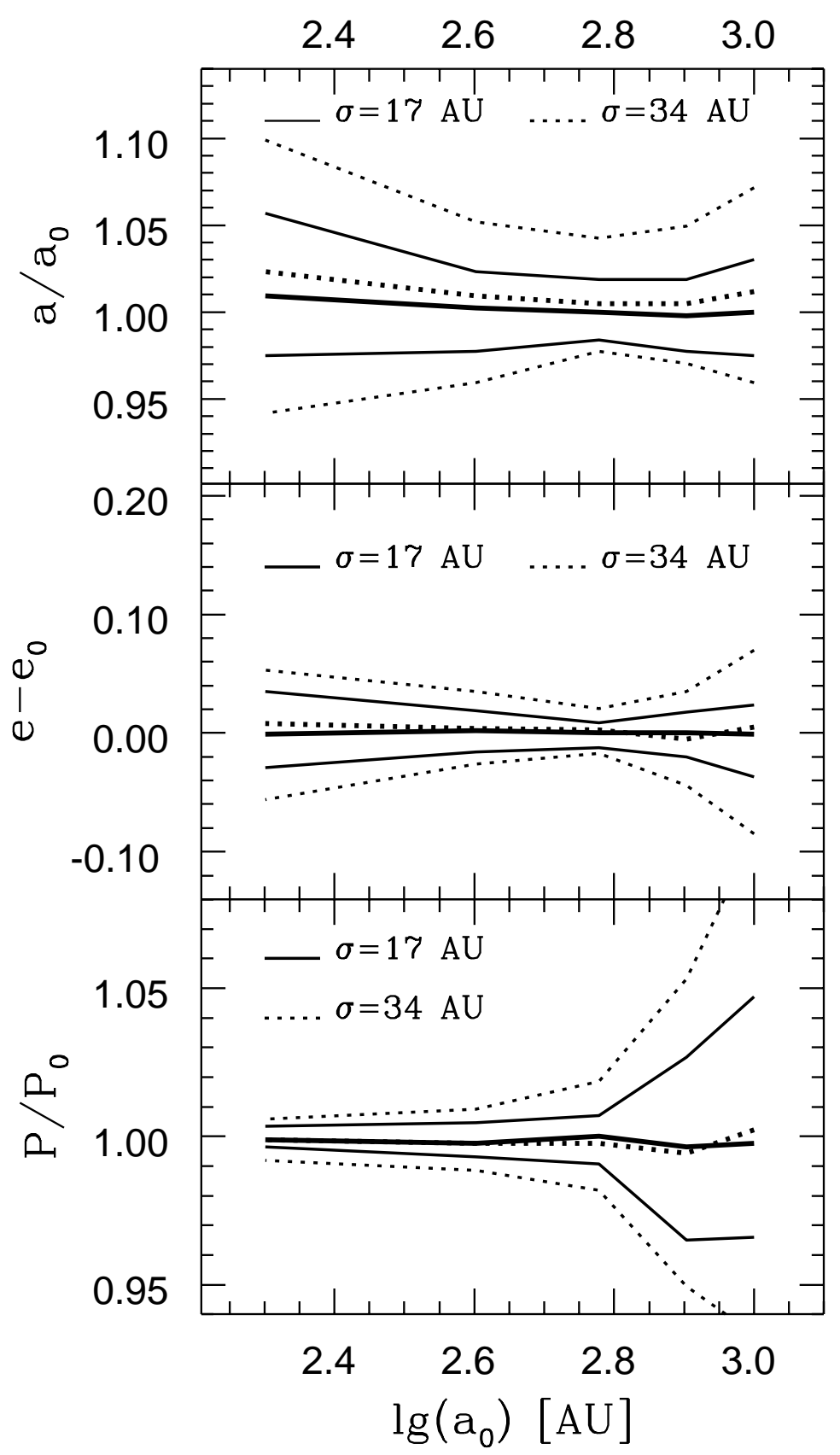




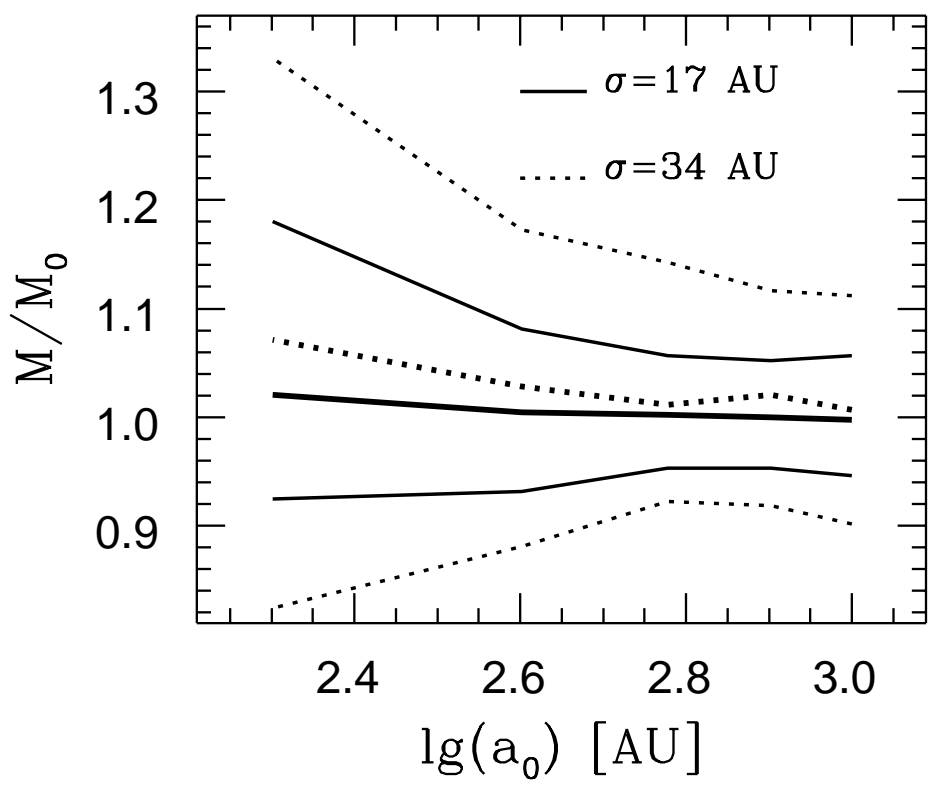




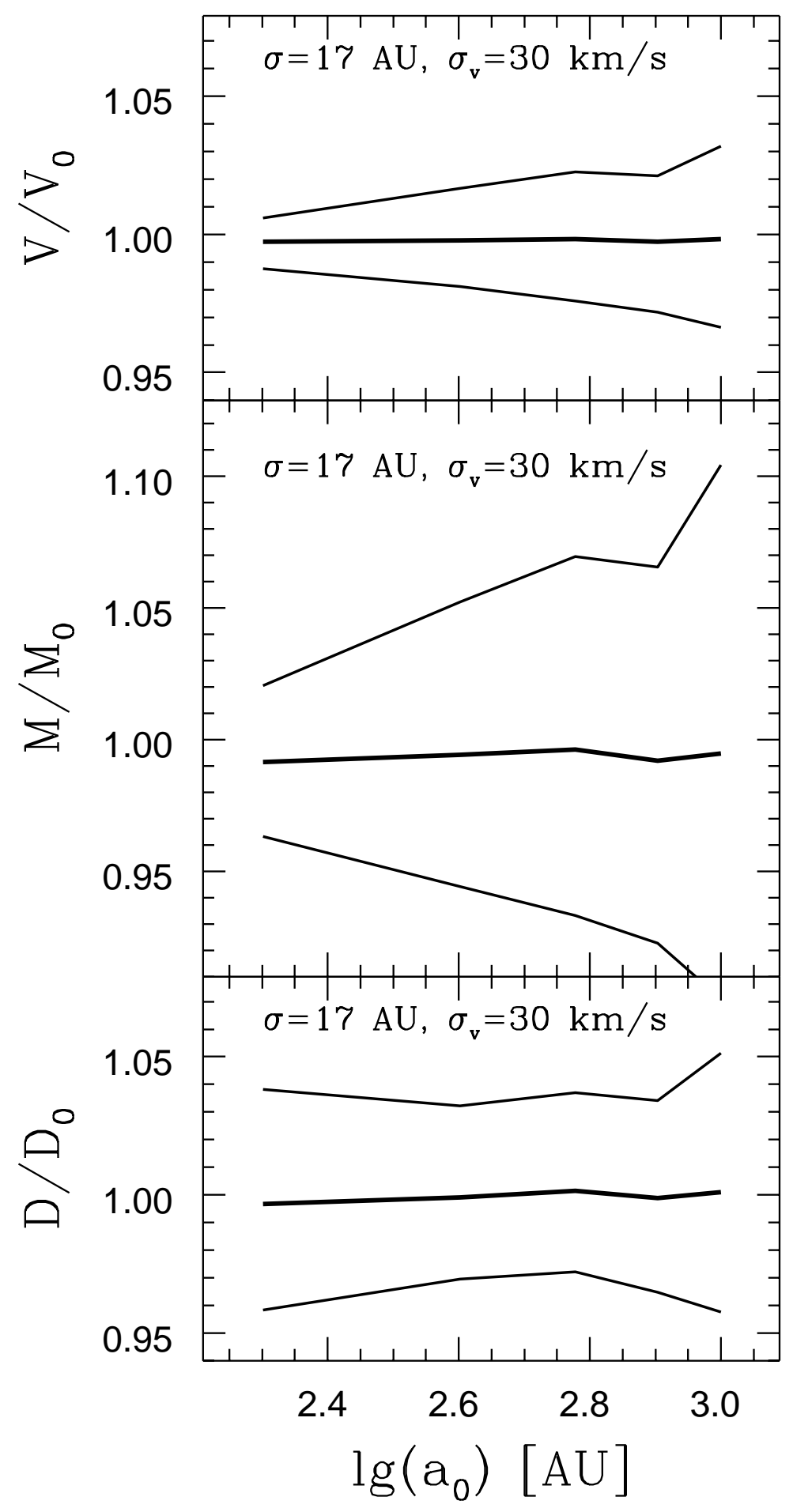




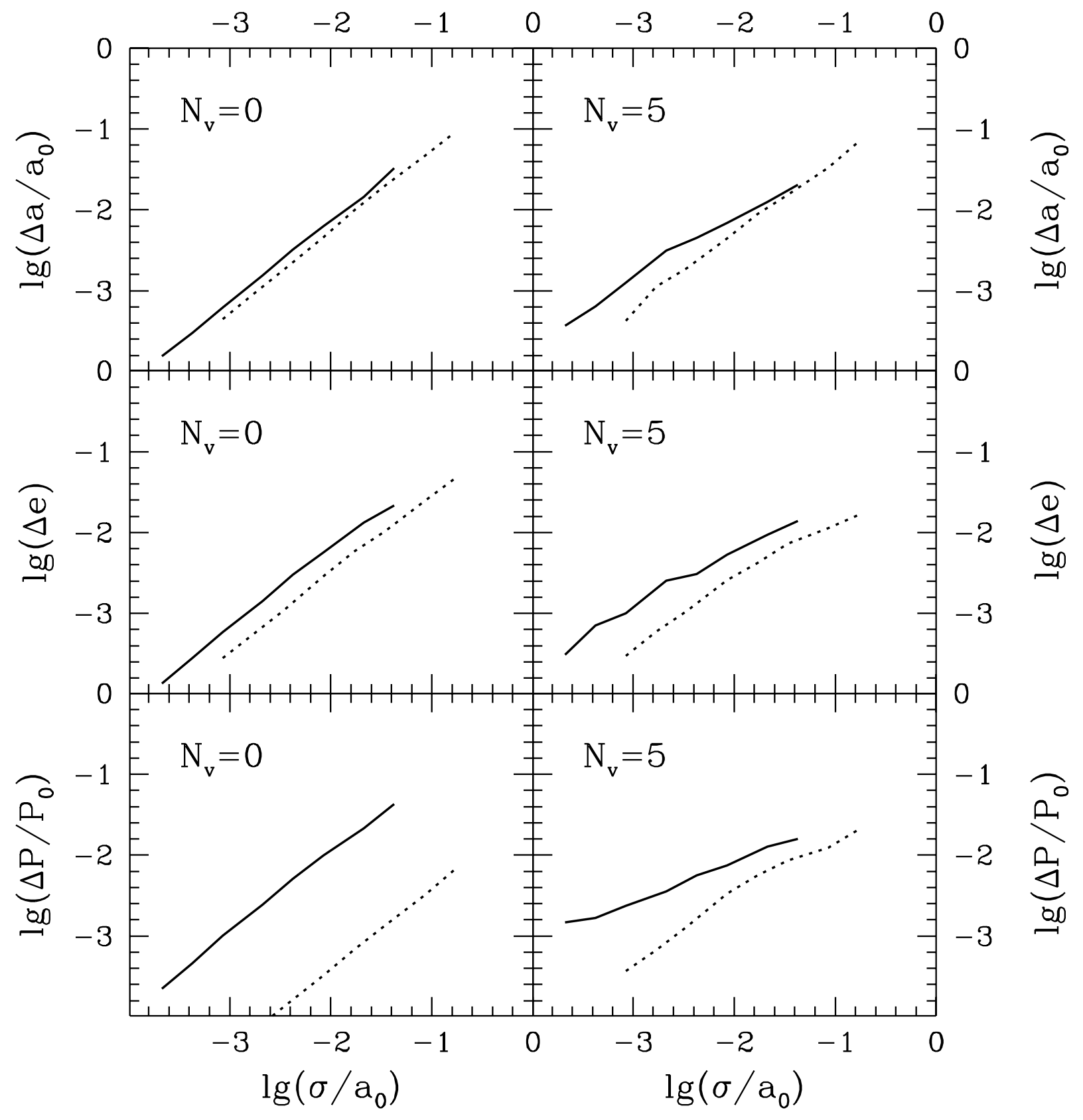




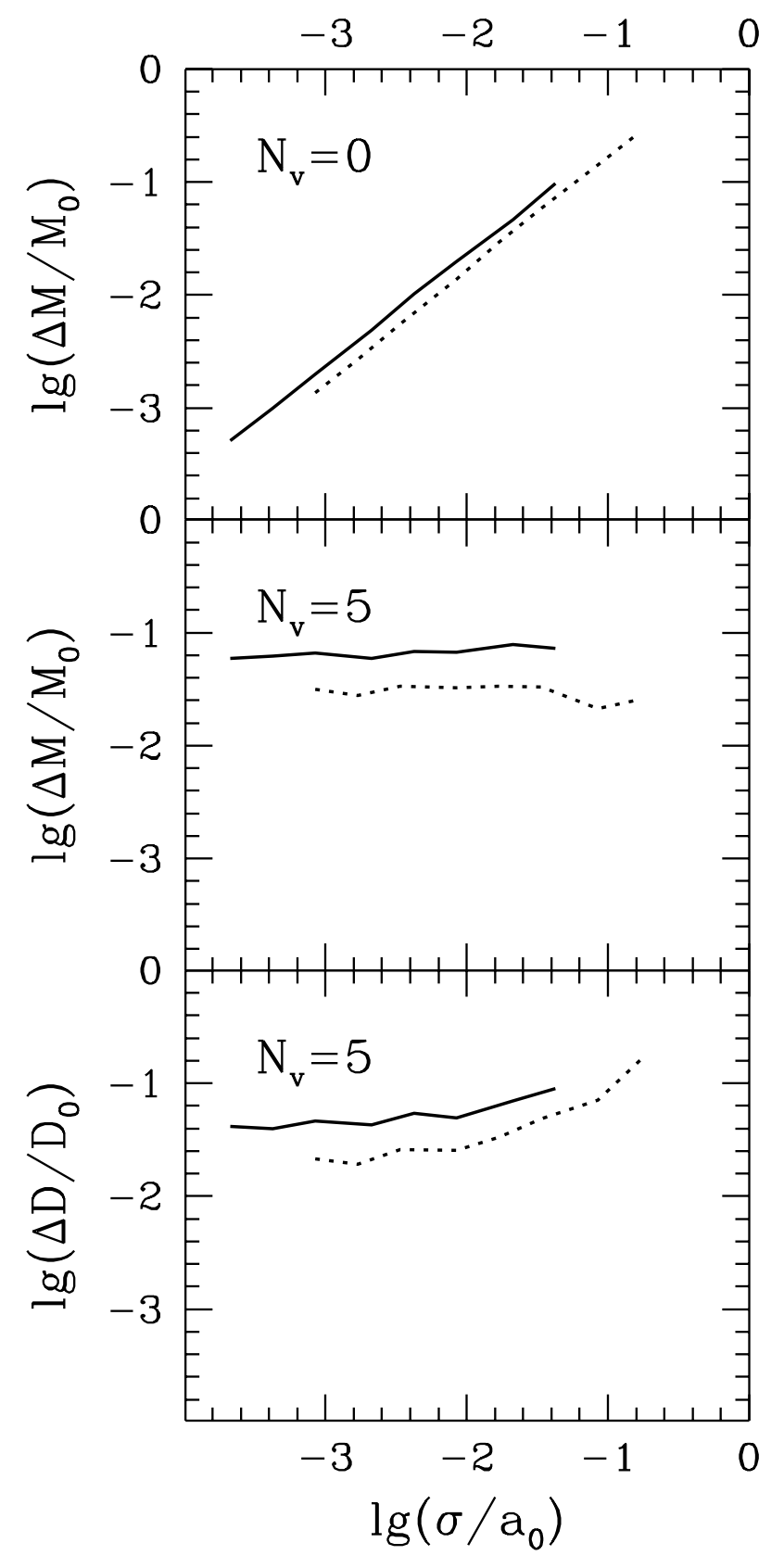

